\title{
Die Medizinisch-ethischen Richtlinien der SAMW aus juristischer Sicht*
}

\author{
D. Rüetschi
}

Das Fehlen einer konkreten gesetzlichen Regelung führt in vielen Gebieten der medizinischen Tätigkeit zu einer erheblichen Rechtsunsicherheit. Die von der SAMW ausgearbeiteten medizinisch-ethischen Richtlinien haben deshalb über die Ärzteschaft hinaus breiten Anklang gefunden. So misst etwa auch das Bundesgericht den Richtlinien bei der Beurteilung medizinrechtlicher Sachverhalte grosse Bedeutung zu. Die Richtlinien haben zwar grundsätzlich keine Gesetzeskraft; durch Verweise in kantonalen Gesetzen erhalten sie aber dennoch eine beschränkte normative Verbindlichkeit. Da sich die Richtlinien mit grundsätzlichen Wertentscheidungen befassen und regelmässig Grundrechte tangieren, sind solche Verweisungen aus rechtsstaatlicher Sicht aber äusserst bedenklich.

Der Konflikt der gegenwärtigen Situation mit den fundamentalen Grundsätzen unseres Rechtsstaates und die positive Aufnahme der Richtlinien durch die Praxis offenbart ein Dilemma zwischen normativem Gebotensein und faktischer Zweckmässigkeit. Erst in jüngster Zeit hat der Gesetzgeber diese angenommen und versucht, eigene gesetzliche Regelungen zu schaffen, wobei die Erfahrung mit den Richtlinien dabei wertvolle Dienste leistet.

\footnotetext{
* Dieser Beitrag ist die überarbeitete und stark gekürzte Fassung eines Papers, welches der Autor im September 2002 an der «Tagung Junger Zivilrechtswissenschaftler» in Heidelberg präsentiert hat und das Interessenten auf Wunsch gerne zugeschickt wird.
}

Korrespondenz:

Dr. iur. David Rüetschi

Handelsgericht des Kantons Aargau

Obere Vorstadt 37

CH-5000 Aarau

Tel. 0628353878

E-Mail: david.rueetschi@ag.ch

\section{Ausgangslage}

«Der Arzt hat das legitime Bedürfnis zu wissen, was er zur Heilung eines Patienten tun darf, ohne das Risiko straf- und zivilrechtlicher Haftung laufen zu müssen. Es kann ihm nicht zugemutet werden, dass er im Ungewissen bleibe, bis sich im Rahmen der Straf- oder Zivilrechtsprechung kasuistisch die von ihm geschuldete Sorgfaltspflicht näher konkretisiert hat» [1]. Trotz dieses anerkannten Anliegens der Ärzteschaft sind viele Bereiche der medizinischen Tätigkeit rechtlich nur in sehr allgemeiner Form oder überhaupt nicht geregelt, und auch den Juristen ist es oftmals kaum möglich, verbindliche Aussagen über die Rechtslage zu machen. Diese Rechtsunsicherheit erschwert die tägliche Arbeit mit den Patienten erheblich.

\section{Die Richtlinien der SAMW}

Vernachlässigt der staatliche Gesetzgeber seine Aufgabe auf diese Weise, erstaunt es nicht, dass private Institutionen diese übernehmen und die Rechtsunsicherheit $\mathrm{zu}$ beseitigen versuchen. In diesem Sinne zielen die Richtlinien der SAMW vor allem auf eine Zusammenfassung der gegen- wärtigen Rechtslage unter Einbezug der in der Allgemeinheit sowie bei den Adressaten vorherrschenden ethischen Wertungen. Dem Arzt soll eine Empfehlung erteilt werden, damit er in schwierigen Situationen eine sachgerechte Entscheidung treffen kann und damit vermeidet, dass er mit dem Recht in Konflikt gerät. Gleichzeitig werden durch eine Definition und Veröffentlichung eines Verhaltensstandards die Abläufe transparent und somit der öffentlichen Diskussion zugänglich gemacht; sie bilden als Stimme der Ärzteschaft überdies Diskussionsgrundlage in Hinblick auf zukünftige gesetzliche Regelungen.

Die Richtlinien der SAMW zeichnen sich vor allem durch ihre Flexibilität aus, was eine rasche Reaktion auf gesellschaftliche Veränderungen ermöglicht, was angesichts des gemächlichen Tempos des schweizerischen Gesetzgebungsprozesses bedeutsam sein kann. Aufgrund der Mobilisierung von Fachwissen sowie der Kompetenz und dem Ansehen der für die Ausarbeitung der Richtlinien verantwortlichen Personen und der SAMW sind die Richtlinien ausserdem von einer hohen Akzeptanz getragen, die eine unmittelbare Steigerung der Effektivität der Normen nach sich zieht, die bei staatlichen Normen nicht gewährleistet wäre. Die Privatisierung der Regelungszuständigkeit bedeutet zuletzt eine Entlastung des staatlichen Gesetzgebers. Als Nachteile springen vor allem die geringere staatliche Kontrolle und die fehlende demokratische Legitimation ins Auge; beklagt wird ausserdem die einseitige Vertretung der Interessengruppen.

\section{Anerkennung bei den Adressaten}

Bereits die erste Richtlinie der SAMW «Richtlinie für die Definition und Diagnose des Todes», in der erstmals die Kriterien zur Feststellung sowohl des Herz- als auch des Hirntodes festgehalten wurden, entwickelte sich in kurzer Zeit zum unverzichtbaren Instrument in der ärztlichen Praxis und erhielt von allen Seiten Anerkennung. Aufgrund des durchschlagenden Erfolgs 
sah sich die Akademie veranlasst, weitere Richtlinien auszuarbeiten, die das gesamte Feld der Bioethikdiskussion abdecken; so finden sich heute Richtlinien zur Sterbehilfe, zur Sterilisation, zur Organtransplantation, zur Forschung und zu genetischen Untersuchungen am Menschen usw. [2]. Die Richtlinien dienen heute als allgemeines Orientierungsinstrument für die Ärzte und werden in der Praxis auch befolgt [3].

\section{Verbindlichkeit der Richtlinien?}

Da es sich bei der SAMW um eine Stiftung gemäss Art. 80ff. ZGB handelt und ihr auch keine hoheitlichen Befugnisse übertragen worden sind, stellen die Richtlinien keine eigentlichen Rechtsnormen dar, sondern lediglich Empfehlungen einer privaten Organisation an ihre Mitglieder. Eine eingeschränkte Verbindlichkeit erhalten die Richtlinien dort, wo die massgebliche ärztliche Standesordnung ausdrücklich auf sie verweist, wie dies in Art. 17f. der Standesordnung der FMH für die Sterbehilfe, Organtransplantation, Sterilisation, ärztlich assistierte Fortpflanzung, Forschungs- und genetische Untersuchungen am Menschen geschehen ist; die Richtlinien werden so zum Bestandteil des ärztlichen Standesrechts, und eine Verletzung durch einen Vertreter des Berufsstandes hat zumindest standesinterne Sanktionen zur Folge.

Eine umfassendere Verbindlichkeit erhalten die Richtlinien dort, wo kantonale Gesetze unmittelbar auf sie verweisen, wie dies bei den Richtlinien zur Todesfeststellung, zu den genetischen Untersuchungen am Menschen, zur Sterbehilfe, zur Organtransplantation und bis zum Inkrafttreten des FMedG - zur Fortpflanzungsmedizin in vielen Kantonen geschehen ist. So lautet etwa Art. 1 der Verordnung über Sterbehilfe und Todesfeststellung des Kantons BE:

«Die im Anhang wiedergegebenen Richtlinien der Schweizerischen Akademie der medizinischen Wissenschaften [...] werden für anwendbar erklärt.»

Ähnlich auch § 27 der Verordnung über die Rechte und Pflichten der Patientinnen und Patienten des Kantons SH:

«Für die Todesfeststellung sind die entsprechenden Richtlinien der SAMW massgebend.»

Die Richtlinien werden auf diese Weise zu verbindlichem Verordnungsrecht. Fraglich bleibt hingegen die Zulässigkeit solcher Verweisungen, denn gemäss Art. 164 Abs. 1 der Bundesverfassung sind alle «wichtigen rechtsetzenden Bestimmungen [...] in der Form des Bundesgesetzes» zu erlassen; auch das Bundesgericht erachtet eine Delegation der Gesetzgebungskompetenz nur dann als zulässig, wenn die Rechtsstellung der Bürger nicht schwerwiegend beeinträchtigt wird [4]. Bezüglich der Richtlinien zur Feststellung des Todes hat das Bundesgericht zwar eingeräumt, dass ein Verweis grundsätzlich zulässig sei, da er im konkreten Fall dem Schutz der Grundrechte der Bürgerinnen und Bürger gedient habe [5]. Dagegen betreffen die übrigen Richtlinien Themen von grösster gesellschaftlicher Relevanz und tangieren unmittelbar Grundrechte, etwa bei der Sterbehilfe oder der Fortpflanzungsmedizin; eine Delegation erscheint hier kaum zulässig, die Regelung müsste vielmehr auf der Stufe des formellen Gesetzes stattfinden [6].

\section{Anerkennung durch das Bundesgericht}

Trotz der grundsätzlichen Unverbindlichkeit der Richtlinien behandelt auch das Schweizerische Bundesgericht diese in seinen Entscheiden [7] mit grossem Respekt und hielt fest: «Le Tribunal fédéral a, depuis longtemps, reconnu la légitimité et la pertinence des directives de l'ASSM» [8]. Dabei fanden nicht nur medizintechnische, sondern auch ethische Vorgaben der Richtlinien Eingang in die Rechtsprechung: So war zu beurteilen, ob eine homologe künstliche Insemination bei (verheirateten oder unverheirateten) Paaren «in stabilen Verhältnissen» eine therapeutische Massnahme darstelle, die von der Krankenversicherung zu bezahlen sei. Das Gericht stützte sich dabei auf die in den Richtlinien getroffene Wertentscheidung: Da dort nicht zwischen Ehe- und unverheirateten Paaren unterschieden wurde, kam es zum Schluss, dass keine ethischen Einwände gegen die homologe Insemination vorlägen und die Kosten von der Versicherung zu tragen seien [9]. Auch bei der Beurteilung der Verfassungsmässigkeit bestimmter Techniken der Fortpflanzungsmedizin wurden die Richtlinien aufgrund ihrer materiellen Autorität als Erkenntnisquelle herangezogen [10]. Eine solche Berücksichtigung der Richtlinien schliesst indessen nicht aus, dass einzelne den Richtlinien zugrundeliegende Wertungen verworfen werden, wie dies bei der vorbehaltlosen Garantie auf Wahrung der Anonymität des Samenspenders geschehen ist [11]. 


\section{Einfluss der Richtlinien auf den Gesetzgeber}

Der Gesetzgeber hat in den ersten Jahrzehnten seit der Publikation der ersten Richtlinie freilich kaum Anstrengungen unternommen, die betroffenen Materien durch den Erlass von Gesetzen ordnungsgemäss zu erfassen. Es drängt sich auf, dieses Untätigbleiben zumindest teilweise auf die Richtlinien selbst zurückzuführen. So hat der Bundesrat festgehalten: «Die heutige Rechtslage, in der die Sterbehilfe nur durch die Richtlinien [...] der SAMW geregelt ist, braucht keine Ergänzung» [12]. Auch mehr als 17 Jahre später verweist er bezüglich der Frage der passiven Sterbehilfe immer noch darauf, dass zwar keine ausdrückliche gesetzliche Regelung bestehe, eine entsprechende Beurteilungsgrundlage sich aber den SAMW-Richtlinien entnehmen lasse [13]. Eine solche faktische und dauerhafte Delegation der gesetzgeberischen Tätigkeit an eine private Organisation ist äusserst bedenklich, denn es ist grundsätzlich die Pflicht des Gesetzgebers, Normen zu schaffen, wenn die gesellschaftliche Realität danach ruft. Die SAMW selber sieht sich denn auch nicht als Ersatzgesetzgeberin, sondern wünscht sich, dass die Richtlinien «nach etwa zehn Jahren Bewährungsprobe und politischer Diskussion zu Gesetz werden sollten» [14] und die Richtlinien lediglich provisorischen Charakter hätten, quasi als vorübergehendes Arbeitsinstrument zu betrachten seien [15]. In diesem Sinne wollen die Richtlinien Ersatz, nicht Konkurrenz zum staatlichen Recht sein. Dies äussert sich auch darin, dass sie ausschliesslich dort erlassen wurden, wo keine oder nur ungenügende formal-gesetzliche Regelungen $\mathrm{zu}$ finden sind und - wie beim FMedG - beim Inkrafttreten von solchen zurückgezogen werden.

Die Betrachtung offenbart ein Dilemma zwischen normativem Gebotensein und faktischer Zweckmässigkeit: Die geltende Lösung widerspricht zwar Grundsätzen unseres Rechtsstaates, scheint aber in der Praxis ausgesprochen gut zu funktionieren, da ein verlässlicher Verhaltensmassstab geschaffen worden ist, wie er vom ordentlichen Gesetzgeber nur schwer hätte geschaffen werden können. Jeder Versuch einer nachträglichen Normierung läuft ernsthaft Gefahr, im inhaltlichen Vergleich mit den bestehenden Richtlinien schlechter abzuschneiden.

\section{Ausblick}

In jüngerer Zeit ist unerwartet Bewegung ins Feld des Medizinrechts gekommen, und es zeigen sich ernsthafte Bestrebungen zur Kodifizierung vieler der durch die Richtlinien abgesteckten Bereiche. So bestehen mittlerweile Berichte und Vorentwürfe zu vielen, gegenwärtig noch von Richtlinien erfassten Bereichen, etwa für die Organtransplantation, die Sterbehilfe, die Sterilisation, die Forschung am Menschen sowie die Stammzellenproblematik. Bereits in Kraft getreten ist das FMedG. Bei den Vorbereitungen der Gesetzgebung erweisen sich die jahrelangen praktischen Erfahrungen mit den Richtlinien als wertvolle Basis. Diese finden als einzige nichtstaatliche Erkenntnisquelle regelmässig Erwähnung in den vorbereitenden Berichten [16] und üben damit auch auf die zu erlassenden Gesetze einen massgeblichen Einfluss aus. Eine parlamentarische Initiative aus dem Jahr 2001 fordert sogar dazu auf, die «bestehenden Gesetzeslücken betreffend die indirekte aktive Sterbehilfe und die passive Sterbehilfe nach den Richtlinien der SAMW zu regeln» [17]. Auch die Regelung des Todeszeitpunktes soll nach Ansicht des Bundesrates «analog den Richtlinien» geregelt werden [18]. Damit werden die Richtlinien auf ihre ursprünglich intendierte Funktion zurück geführt: Sie sollen erstens als Übergangslösung dienen und zweitens dazu beitragen, eine Erfahrungsgrundlage für spätere Gesetze zu schaffen.

\section{Literatur}

1 Müller JP. Recht auf Leben. Persönliche Freiheit und das Problem der Organtransplantation. ZSR 1971;90:457-78.

2 Sämtliche Richtlinien sind abrufbar unter www.samw.ch.

3 Vgl. nur Jaag T, Rüssli M. Sterbehilfe in staatlichen Spitälern, Kranken- und Altersheimen. ZBl 2001;102:113-29; auch die von Heine G in: Eser A, Koch HG. Materialien zur Sterbehilfe. Freiburg 1999;591-649, durchgeführte Umfrage bei Behörden, Ärzten und Spitälern kommt zum Ergebnis, dass den Sterbehilferichtlinien in der Praxis erhebliche Orientierungsfunktion zukommt; vgl. auch Weibel ER. Von der Ethik der Transplantationsmedizin. NZZ, 12.1.1999, wonach bis heute kein Verstoss gegen die Richtlinien bekannt sei.

4 Vgl. BGE 104 Ia 310.

5 Vgl. BGE 123 I 130

6 In diesem Sinne hat das Bundesgericht zumindest bezüglich der Zulassung unverheirateter Paare zur Fortpflanzungsmedizin entschieden, vgl. Urteil vom 26.10.1989, plädoyer 1990;2:60-2. 
7 Vgl. BGE 98 Ia 508ff.; 123 I 112ff. (beide Todesfeststellung), Urteil von 26.10.1989, plädoyer 1990;2:60-2; BGE 115 Ia 234ff.; 119 Ia 460ff.; 121 V 289ff.; 121 V 302ff. (alle Fortpflanzungsmedizin).

8 BGE 123 I 128.

9 BGE 121 V 301; bestätigt in 121 V 310f.; anders noch BGE $119 \mathrm{~V}$ 26ff. und Verwaltungsgericht ZH, ZBl 2000;101:489-96, welches die Sterbehilferichtlinie nur als «ethisch motivierte Empfehlungen» ohne medizinwissenschaftliche Begründung betrachtete.

10 BGE 115 Ia 267; 119 Ia 483 (Zulässigkeit der heterologen Insemination); 115 Ia $251 \mathrm{ff}$ (Anonymität des Samenspenders); 119 Ia 503 (Verwendung menschlicher Embryonen zu Forschungszwecken).

11 Vgl. BGE 115 Ia 256.

12 Botschaft zur Volksinitiative «Recht auf Leben», BBl 1983 II 27; vgl. auch die Antwort des Bundesrates zur Interpellation Segmüller, BBl. 1985, 1813, wo das Bedürfnis nach Normierung der Forschung an Embryonen mit Verweis auf die Richtlinien der SAMW verneint wurde.

13 Antwort des Bundesrates auf die Interpellation Waber, AB NR 2001 (Beil.), 344.
14 Hitzig. BIOTECHforum 1998;1:6-7.

15 Vgl. Courvoisier B. Jahresbericht SAMW. Basel 1991:50.

16 Botschaft zur Volksinitiative «Recht auf Leben», BBl 1983 II 27; Bericht des Bundesamts für Justiz zu den Patiententestamenten, VPB 1987, Nr. 44; Botschaft über die Volksinitiative «zum Schutz des Menschen vor Manipulationen in der Fortpflanzungstechnologie» und zu einem Bundesgesetz über die medizinisch unterstützte Fortpflanzung, BBI 1996 III 209f.; Begleitbericht zum Vorentwurf für ein Bundesgesetz über genetische Untersuchungen beim Menschen, Bern 1998 , 25, 27; Arbeitsgruppe Sterbehilfe. Bericht der Arbeitsgruppe an das EJPD. März 1999, 12ff.; Bericht der Rechtskommission des Nationalrates zur parlamentarischen Initiative Cavalli, AB NR 2001 (Beil.), 95f.; Antwort des Bundesrates auf die Interpellation Waber, AB NR 2001 (Beil.), 344; Botschaft zum Bundesgesetz über die Transplantation von Organen, Geweben und Zellen, BBl 2002, 29, 103; Botschaft zum Bundesgesetz über genetische Untersuchungen beim Menschen, BBl 2002, 7374.

17 Vgl. Motion Zäch, AB NR 2001 (Beil.), 420.

18 Vgl. Botschaft TPG, BBl 2002, 140. 